\title{
Observation of the Superconducting Proximity Effect in the Surface State of $\mathrm{SmB}_{6}$ Thin Films
}

\author{
Seunghun Lee, ${ }^{1,2}$ Xiaohang Zhang, ${ }^{1,2}$ Yangang Liang, ${ }^{1,2}$ Sean W. Fackler, ${ }^{1,2}$ Jie Yong, ${ }^{2}$ Xiangfeng Wang, ${ }^{2}$ \\ Johnpierre Paglione, ${ }^{2}$ Richard L. Greene, ${ }^{2}$ and Ichiro Takeuchi ${ }^{1,2,}$ \\ ${ }^{1}$ Department of Materials Science and Engineering, University of Maryland, \\ College Park, Maryland 20742, USA \\ ${ }^{2}$ Center for Nanophysics and Advanced Materials, Department of Physics, University of Maryland, \\ College Park, Maryland 20742, USA \\ (Received 7 April 2016; revised manuscript received 19 June 2016; published 25 August 2016)
}

\begin{abstract}
The proximity effect at the interface between a topological insulator and a superconductor is predicted to give rise to chiral topological superconductivity and Majorana fermion excitations. In most topological insulators studied to date, however, the conducting bulk states have overwhelmed the transport properties and precluded the investigation of the interplay of the topological surface state and Cooper pairs. Here, we demonstrate the superconducting proximity effect in the surface state of $\mathrm{SmB}_{6}$ thin films which display bulk insulation at low temperatures. The Fermi velocity in the surface state deduced from the proximity effect is found to be as large as $10^{5} \mathrm{~m} / \mathrm{s}$, in good agreement with the value obtained from a separate transport measurement. We show that high transparency between the topological insulator and a superconductor is crucial for the proximity effect. The finding here opens the door to investigation of exotic quantum phenomena using all-thin-film multilayers with high-transparency interfaces.
\end{abstract}

DOI: 10.1103/PhysRevX.6.031031

\section{INTRODUCTION}

Topological insulators (TI), which show exotic metallic surfaces with insulating bulk, have attracted tremendous interest in the condensed matter physics community. The topologically protected surface state of TIs has been regarded as a promising platform for exploring exotic quantum phenomena in solid materials. In particular, it has been suggested that the superconducting proximity effect occurring at an interface between a TI and a superconductor may give rise to chiral topological superconductivity [1-3] and Majorana fermion excitations [4-9]. To this end, concerted experimental efforts have been made to study the superconducting proximity effect in superconductor-TI bilayer structures. However, in most $\mathrm{Bi}$ - and Te-based Tis, such as $\mathrm{Bi}_{2} \mathrm{Se}_{3}, \mathrm{Bi}_{2} \mathrm{Te}_{3}, \mathrm{Sb}_{2} \mathrm{Te}$, etc., the overwhelming conducting bulk electronic states hinder interfacial quantum phenomena and preclude the investigation of the interplay of the topological surface state and Cooper pairs [10-13]. In addition, low transparency at the superconductor-TI interface due to a nonpristine surface of the TI can significantly

\footnotetext{
* Corresponding author. takeuchi@umd.edu

Published by the American Physical Society under the terms of the Creative Commons Attribution 3.0 License. Further distribution of this work must maintain attribution to the author(s) and the published article's title, journal citation, and DOI.
}

Subject Areas: Condensed Matter Physics,

Superconductivity,

Topological Insulators reduce the extent of the proximity effect [5,14-16]. Therefore, suppressing the bulk conductivity and securing high interfacial transparency in superconductor-TI bilayers have been an outstanding issue in this field.

Samarium hexaboride $\left(\mathrm{SmB}_{6}\right)$ has recently emerged as an ideal material to explore TI-based quantum phenomena because of its true insulating bulk [17]. To date, many theoretical and experimental studies have provided strong evidence for the existence of the conducting surface state and a robust insulating bulk of $\mathrm{SmB}_{6}$ at low temperatures [18-38]. Specifically, electronic transport measurements on $\mathrm{SmB}_{6}$ single crystals have revealed surface-currentdominated conduction [20,31] and thickness-independent Hall voltage at low temperatures due to the conducting surface [20]. A point contact spectroscopy study has indicated the presence of an insulating bulk in $\mathrm{SmB}_{6}$ at low temperatures $[23,39]$.

Even though there still remain questions regarding the nature of the surface state of $\mathrm{SmB}_{6}$, including the origin of the metallic surface state and whether the surface state is truly topologically protected [17,22,27,40-42], recent angle-resolved photoemission spectroscopy (ARPES) and quantum oscillation experiments have provided strong evidence in support of the predicted nontrivial topological nature of the surface state in $\mathrm{SmB}_{6}[19,32-34]$. In particular, a circular dichroism APRES measurement has demonstrated the spin-polarized surface state of $\mathrm{SmB}_{6}$ as a signature of a topologically protected surface state 
$[32,34]$. We have previously demonstrated successful fabrication of $\mathrm{SmB}_{6}$ thin films using a cosputtering method [37]. The electronic transport properties of the $\mathrm{SmB}_{6}$ thin films were found to be consistent with those of single crystalline $\mathrm{SmB}_{6}$, and they support the topological Kondo insulator picture of $\mathrm{SmB}_{6}[36,37]$.

In this paper, we demonstrate direct observation of the proximity effect induced in the surface state of $\mathrm{SmB}_{6}$ for the first time. We apply the Usadel formalism to the systematic change in the superconducting transition temperature of a series of in situ deposited $\mathrm{SmB}_{6} / \mathrm{Nb}$ bilayers with different $\mathrm{Nb}$ layer thicknesses and arrive at the normal coherence length of the surface state of $\mathrm{SmB}_{6}$ to be $\approx 9 \mathrm{~nm}$. From a separate transport study on $\mathrm{SmB}_{6}$ thin films, we find the thickness of the surface state to be $\approx 6 \mathrm{~nm}$. The Fermi velocity $\left(v_{F}\right)$ obtained from the transport measurements is in good agreement with $v_{F}$ calculated using the normal coherence length, and it is of the order of $10^{5} \mathrm{~m} / \mathrm{s}$. These numbers paint a picture of a material whose bulk insulation provides a unique opportunity to probe and exploit its surface state in thin-film multilayer devices.

\section{METHODS}

$\mathrm{SmB}_{6}$ thin films are prepared by cosputtering of $\mathrm{SmB}_{6}$ and $\mathrm{B}$ targets; additional $\mathrm{B}$ sputtering is performed for compensating B deficiency, and the base pressure is $\sim 2 \times 10^{-8}$ Torr. The thin films are grown on a Si (100) substrate at $800^{\circ} \mathrm{C}$ in an atmosphere of $\operatorname{Ar}(99.999 \%)$ and the working pressure is kept at 10 mTorr. After deposition, further annealing is performed at $800{ }^{\circ} \mathrm{C}$ for $3 \mathrm{~h}$ in highvacuum. X-ray diffraction and transmission electron microscopy results can be found in Ref. [37]. For fabricating $\mathrm{Nb} / \mathrm{SmB}_{6}$ bilayers, $\mathrm{Nb}$ layers are prepared on $\mathrm{SmB}_{6}$ by the sputtering method both in situ and ex situ at room temperature. The base pressure is $\sim 2 \times 10^{-8}$ Torr and the working pressure is kept at 7 mTorr. For fabricating the $\mathrm{Nb} / \mathrm{Au}$ bilayer, a Au layer is prepared on the Si substrate by a thermal evaporator and the base pressure is $\sim 10^{-6}$ Torr.

The electrical properties are measured by a physical properties measurement system. The temperaturedependent resistance for $T_{c}$ evaluation is measured by a simple four-point probe method with $0.01 \mathrm{~K}$ step. Electrical contacts are made with an $\mathrm{Al}$ wire bonder, and the typical contact resistance is $\sim 1 \Omega$. Transport characteristics of $\mathrm{SmB}_{6}$ thin films are measured with Hall bar geometry; six-contact 1-2-2-1 Hall bars with $200-\mu \mathrm{m}$ channel size are prepared by an ion milling process.

\section{RESULTS AND DISCUSSION}

\section{A. Transport characteristics}

We perform transport measurements on $\mathrm{SmB}_{6}$ thin films with varying film thickness. In order to perform the transport measurements, each film is patterned into a Hall bar with a channel width of $200 \mu \mathrm{m}$. Resistance

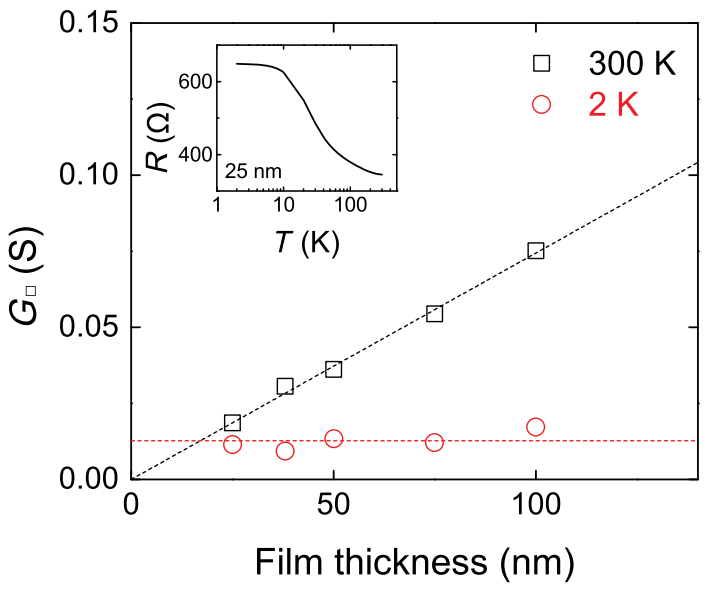

FIG. 1. Sheet conductance $\left(G_{\square}\right)$ of $\mathrm{SmB}_{6}$ thin films at 2 and $300 \mathrm{~K}$ as a function of film thickness. Dashed lines are linear fits to the experimental results. The slope of $300 \mathrm{~K}$ data represents $3 \mathrm{D}$ bulk conductivity at $300 \mathrm{~K}$ in $\mathrm{SmB}_{6}$ thin films, and the fit to the data (black dot line) extrapolates to zero at zero thickness, consistent with the fact that the conduction in $\mathrm{SmB}_{6}$ thin films is dominated by a $3 \mathrm{D}$ bulk transport at $300 \mathrm{~K}$. At $2 \mathrm{~K}, G_{\square}$ is independent of the thickness, and the guide line (red dotted line) is the fit to the data, which indicates the average value of $G_{\square}$ at $2 \mathrm{~K}$. The inset shows resistance versus temperature curve for a $25-\mathrm{nm} \mathrm{SmB}_{6}$ thin film.

versus temperature $(R-T)$ is measured for all $\mathrm{SmB}_{6}$ films, and their sheet conductance $\left(G_{\square}\right)$ values at 300 and $2 \mathrm{~K}$ are plotted in Fig. 1. $G_{\square}$ is calculated using $G_{\square}=G \cdot a / w$, where $a$ and $w$ are the length and the width of the Hall bar channels, respectively. All samples show a nearly constant resistance value at low temperatures. The inset shows a representative $R-T$ curve for a $25-\mathrm{nm}$ thin film. The result is consistent with previous reports [24,29,31,35] where a saturation of resistance (i.e., plateau) has been attributed to the transition from bulk-state-dominated conduction to surface-state-dominated conduction at low temperatures.

To see if there is any thickness-dependent effect in our samples, we plot $G_{\square}$ at 2 and $300 \mathrm{~K}$ as a function of film thickness (Fig. 1). $G_{\square}$ at $300 \mathrm{~K}$ clearly displays a linear thickness dependence, indicating bulk transport. However, if the resistance plateau at low temperatures arises from the surface conduction, $G_{\square}$ at $2 \mathrm{~K}$ should be independent of the thickness and become a constant. This is indeed the case, as shown in Fig. 1, strongly suggestive of the presence of the surface conduction channel. We also measure the Hall resistance $\left(R_{x y}\right)$ as a function of magnetic field, and calculate the sheet carrier concentration and the mobility of the $\mathrm{SmB}_{6}$ thin films at $2 \mathrm{~K}$. Both are found to be constant and independent of the film thickness (see Supplemental Material [43]).

To determine the thickness of the surface conduction channel, we adopt a simple parallel conductance model where the electronic conduction is through two channels: the surface channel including contributions from both the 
top and the bottom surfaces and the bulk channel $[24,31,44]$. This model is valid for the temperature region where the Kondo gap is fully open, which is a sufficient condition for the presence of the surface state. Thus, we apply this model to the transport results in the temperature range below $30 \mathrm{~K}$, where the Kondo hybridization gap has been reported to fully open in $\mathrm{SmB}_{6}[23,25,45,46]$. The conductance of the surface channel is assumed to be temperature independent, and that of the bulk channel is temperature dependent and it can be described by a bulk resistivity and exponential function (i.e., Arrhenius equation). The total conductance of the $\mathrm{SmB}_{6}$ thin film can then be described as

$$
\begin{gathered}
G=G_{\text {surface }}+G_{\text {bulk }}, \\
G_{\text {surface }}=\frac{w}{a} G_{\square, 2 \mathrm{~K}}, \\
G_{\text {bulk }}=\frac{w \cdot t_{\text {bulk }}}{a} \cdot \sigma_{\text {bulk }, 300 \mathrm{~K}} \exp \left(-\frac{E_{a}}{k_{B} T}+\frac{E_{a}}{300 k_{B}}\right) .
\end{gathered}
$$

The use of the bulk conductivity in the $G_{\text {bulk }}$ term allows us to add a thickness of bulk channel $\left(t_{\text {bulk }}\right)$ as fitting parameters, and subsequently to estimate a thickness of surface channel $\left(t_{\text {surface }}\right)$ because the total film thickness is $2 t_{\text {surface }}+t_{\text {bulk }}$. The bulk conductivity $\sigma_{\text {bulk,300 K }}$ is $7.4 \times 10^{5} \mathrm{~S} \mathrm{~m}^{-1}$, which is the slope of $G_{\square}$ at $300 \mathrm{~K}$ obtained from the data shown in Fig. 1. $G_{\square, 2 \mathrm{~K}}$ is the $G_{\square}$ at $2 \mathrm{~K}$ for each sample, and $a$ and $w$ are the length and the width of the Hall bar channel, respectively. Fitting parameters are $t_{\text {bulk }}$ and $E_{a}$, where $E_{a}$ is an activation energy. We fit the data for all samples using Eqs. (1)-(3). A representative fitting result is shown in Fig. 2(a). The values of fitting parameters $E_{a}$ and $t_{\text {surface }}$ for the different thickness samples are shown in Fig. 2(b), and $t_{\text {surface }}$ is the thickness of the surface conduction channel, i.e., the thickness of the surface state of $\mathrm{SmB}_{6}$. Both $E_{a}$ and $t_{\text {surface }}$ are found to be constant regardless of the film thickness. The average value of $E_{a}$ is, $3.1 \pm 0.1 \mathrm{meV}$, which is in good agreement with the values obtained from $\mathrm{SmB}_{6}$ single crystals [24,28,31].

From the above analysis, we obtain $t_{\text {surface }} \approx 6 \mathrm{~nm}$. To obtain $v_{F}$, we use $v_{F}=E_{g} t_{\text {surface }} / h$ derived from the solution of the effective low-energy Hamlitonian for topological insulators with a surface state where $E_{g}$ is the bulk energy gap [29,47]. We note that $E_{a}$ obtained from transport measurements does not correspond to the intrinsic hybridization gap of $\mathrm{SmB}_{6}$, because the activation energy related to the transport characteristics of the bulk state of $\mathrm{SmB}_{6}$ may be affected by the presence of residual states in the gap or other activation behaviors $[39,46]$. Instead, we use $E_{g}$ of $17 \mathrm{meV}$ measured on our $\mathrm{SmB}_{6}$ thin films by terahertz spectroscopy [48], which is in good agreement with $E_{g}$ values obtained from single crystal $\mathrm{SmB}_{6}$ using point contact spectroscopy [23], scanning tunneling spectroscopy [25], etc. With $E_{g}=17 \mathrm{meV}$ and $t_{\text {surface }}=6 \mathrm{~nm}$, we arrive at $v_{F} \sim 1.6 \times 10^{5} \mathrm{~m} / \mathrm{s}$, a value close to that measured in quantum oscillation measurements on single crystal $\mathrm{SmB}_{6}$ [19]. This value is also in agreement with an independent estimate of $v_{F}$ obtained from the proximity effect study as discussed below.

\section{B. Proximity effect}

The superconducting proximity effect describes a phenomenon at a superconductor-normal metal interface where Cooper pairs diffuse into the normal metal resulting in the suppression of the critical temperature $\left(T_{c}\right)$ of the superconductor while inducing surface or local superconductivity in the normal metal. We fabricate $\mathrm{Nb} / \mathrm{SmB}_{6}$ bilayers and observe a change in $T_{c}$ depending on the thickness of the $\mathrm{Nb}$ layer due to the proximity effect at the interface. To characterize the proximity effect of the bilayers, we treat
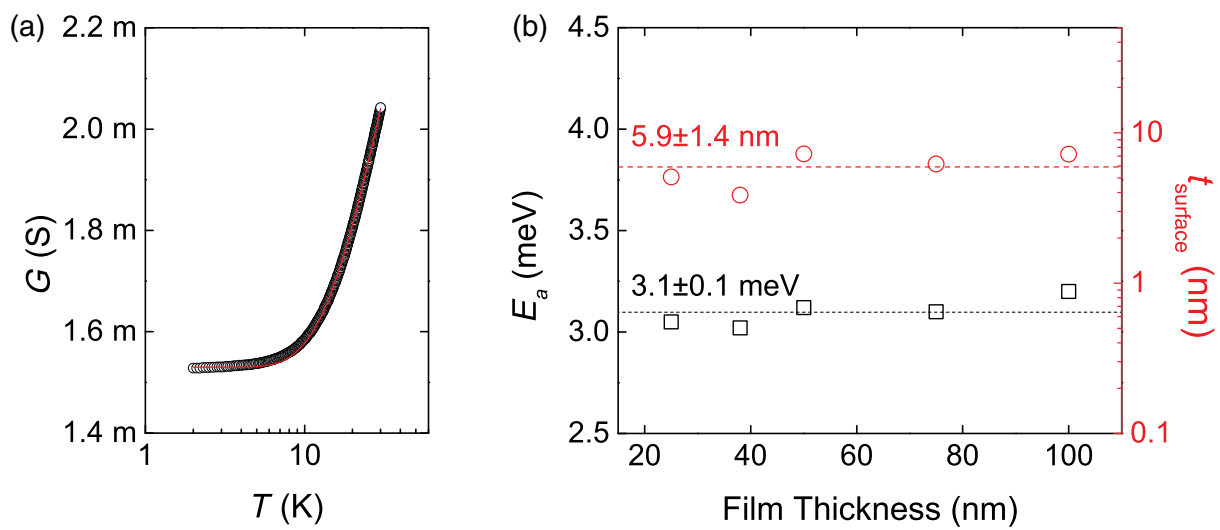

FIG. 2. Estimating the thickness of the surface conduction channel in $\mathrm{SmB}_{6}$ thin films. (a) Conductance versus temperature of a 25 -nm $\mathrm{SmB}_{6}$ thin film. The red line is a best fit to the experimental data using the parallel conductance model. (b) Activation energy $\left(E_{a}\right)$ and the thickness of the surface conduction channel ( $\left.t_{\text {surface }}\right)$ extracted from the fit as a function of the film thickness for $\mathrm{SmB}_{6}$. 


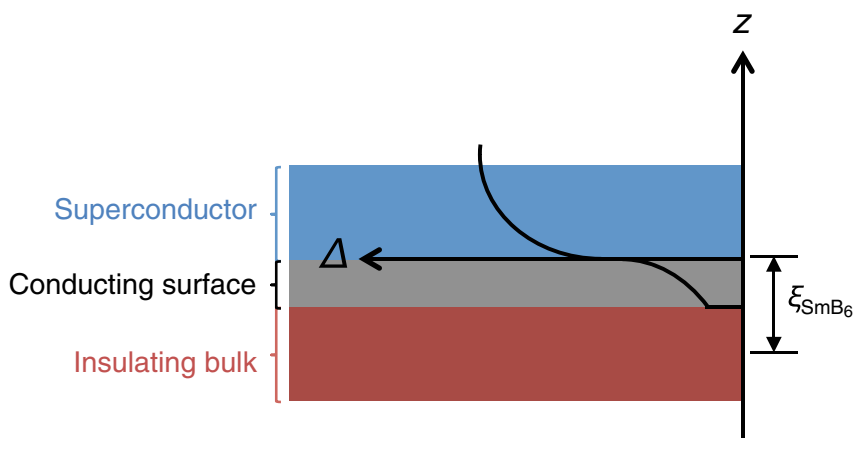

FIG. 3. Model for the proximity effect for superconducting-TI system. Given the surface conducting channel of the $\mathrm{SmB}_{6}$ as discussed in the text, we adopt the model of the proximity effect for the superconducting layer and the surface conduction channel, the surface state of $\mathrm{SmB}_{6}$. The plot in the schematic represents the evolution of the superconducting pair potential $[\Delta(\mathrm{z})]$. In this model, we consider the case where the normal coherence length of $\mathrm{SmB}_{6}\left(\xi_{\mathrm{SmB}_{6}}\right)$ is larger than the thickness of the surface state of $\mathrm{SmB}_{6}\left(t_{\text {surface }}\right)$.

the $\mathrm{Nb} / \mathrm{SmB}_{6}$ bilayer as a superconductor-metal bilayer system, where the metallic layer in $\mathrm{SmB}_{6}$ is the surface conducting channel (Fig. 3), and we calculate the normal coherence length and $v_{F}$ of $\mathrm{SmB}_{6}$ thin films using the Usadel equation $[16,49,50]$. Assuming that the normal coherence length of $\mathrm{SmB}_{6}\left(\xi_{\mathrm{SmB}_{6}}\right)$ is longer than the thickness of the surface conducting channel of $\mathrm{SmB}_{6}$ $\left(\xi_{\mathrm{SmB}_{6}}>t_{\text {surface }}\right)$, the fitting equation for evaluating $\xi_{\mathrm{SmB}_{6}}$ can be obtained by linearizing the Usadel equation [16]:

$$
\begin{gathered}
\frac{T_{c b}}{T_{c s}}=1-\frac{\pi^{2}}{4} \frac{\xi_{\mathrm{Nb}}}{d_{\mathrm{Nb}}} \gamma \cdot p(\gamma), \\
\gamma=\frac{\rho_{\mathrm{Nb}} \xi_{\mathrm{Nb}} t_{\text {surface }}}{\rho_{\mathrm{SmB}_{6}}\left(\xi_{\mathrm{SmB}_{6}}\right)^{2}}, \\
p(\gamma)=1.17+\frac{2}{\pi^{2}} \ln \left(1+0.98 \gamma^{-2}\right),
\end{gathered}
$$

where $T_{c b}$ and $T_{c s}$ represent the $T_{c}$ of the $\mathrm{Nb} / \mathrm{SmB}_{6}$ bilayer and the $T_{c}$ of a single $\mathrm{Nb}$ layer, respectively. $T_{c b}$ is evaluated by the Ginzburg-Landau equation, and $\gamma$ and $p(\gamma)$ are approximated by the expressions above [16]. $\gamma$ represents the strength of the proximity effect between layers [49]. $\rho_{\mathrm{Nb}}$ and $\rho_{\mathrm{SmB}_{6}}$ are the residual resistivities of the $\mathrm{Nb}$ layer and the $\mathrm{SmB}_{6}$ layer, respectively. $\xi_{\mathrm{Nb}}$ is the superconducting coherence length of the $\mathrm{Nb}$ layer. $d_{\mathrm{Nb}}$ is the thickness of the $\mathrm{Nb}$ layer. To check the validity of the model, a series of $\mathrm{Nb} / \mathrm{Au}$ bilayers are also fabricated, and the extracted value of normal coherence length of $\mathrm{Au}\left(\xi_{\mathrm{Au}}\right)$ is found to be in the range of its previously reported values [51,52], and it is also consistent with the clean limit value of $\xi_{\mathrm{Au}}$.

For $\mathrm{Nb} / \mathrm{SmB}_{6}$ bilayers, $d_{\mathrm{Nb}}$ is varied from 20 to $100 \mathrm{~nm}$, and different thicknesses of $\mathrm{SmB}_{6}$ layers $(10,25$, and (a)

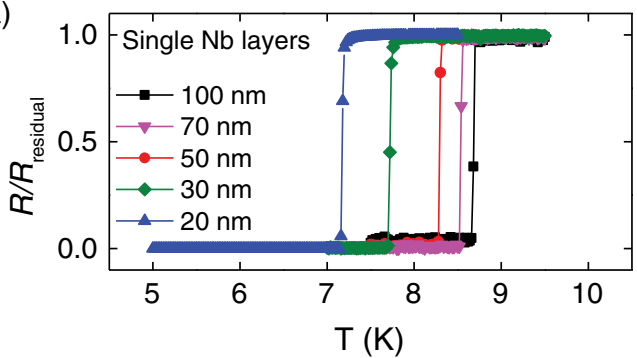

(b)
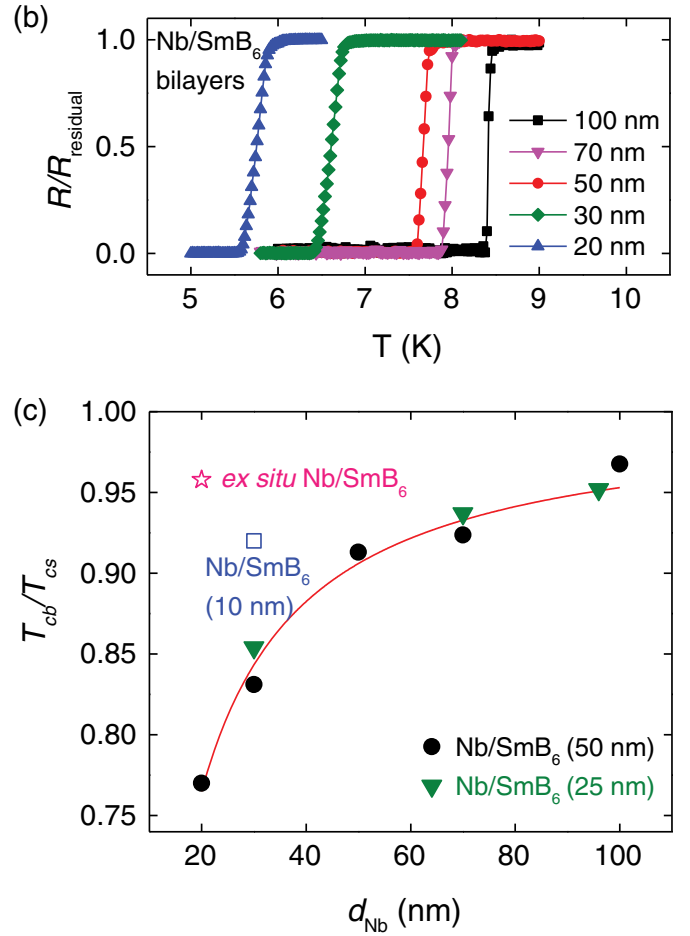

FIG. 4. Proximity effect in $\mathrm{Nb} / \mathrm{SmB}_{6}$ bilayer. Resistance versus temperature curves of (a) single $\mathrm{Nb}$ layers and (b) $\mathrm{Nb} / \mathrm{SmB}_{6}$ bilayers for different $\mathrm{Nb}$ layer thicknesses $\left(d_{\mathrm{Nb}}\right)$ where the $\mathrm{SmB}_{6}$ layer thickness is fixed to be $50 \mathrm{~nm}$. Each Nb layer is deposited on $\mathrm{SmB}_{6}$ in situ. The resistance values are normalized by values obtained at temperatures slightly above each transition temperature $\left(R_{\text {residual }}\right)$. (c) $T_{c b} / T_{c s}$ (i.e., ratio of $T_{c}$ of $\mathrm{Nb} / \mathrm{SmB}_{6}$ bilayer to $T_{c}$ of single $\mathrm{Nb}$ layer) as a function of $d_{\mathrm{Nb}}$. The data points from bilayers with different $\mathrm{SmB}_{6}$ layer thicknesses (10 and $25 \mathrm{~nm}$ ) are also included. The solid line is a fit using the Usadel equation. The star indicates $T_{c b} / T_{c s}$ of the $\mathrm{Nb} / \mathrm{SmB}_{6}$ bilayer where $20-\mathrm{nm} \mathrm{Nb}$ is deposited on $\mathrm{SmB}_{6}(50 \mathrm{~nm})$ after its surface is first exposed to air, forming an ex situ interface.

$50 \mathrm{~nm}$ ) are used. Figures 4(a) and 4(b) show the resistance versus temperature curves of single $\mathrm{Nb}$ layers and the bilayers with 50 -nm-thick $\mathrm{SmB}_{6}$ near $T_{c}$ for different $\mathrm{Nb}$ layer thicknesses $\left(d_{\mathrm{Nb}}\right)$, respectively. The resistance values are normalized by values obtained at temperatures slightly above each transition temperature $\left(\mathrm{R}_{\text {residual }}\right)$. The $T_{c}$ of a single $\mathrm{Nb}$ layer gradually decreases with decreased $d_{\mathrm{Nb}}$ due to localization [53], and each $\mathrm{Nb} / \mathrm{SmB}_{6}$ bilayer has a lower $T_{c}$ than the corresponding $\mathrm{Nb}$ single layer due to the proximity effect established between $\mathrm{Nb}$ and $\mathrm{SmB}_{6}$ layers. 
From x-ray diffraction and cross-sectional SEM of the bilayers, no signature of diffusion is detected at the interface. We measure the roughness of the $\mathrm{SmB}_{6}$ layers, and they are found to be very smooth (with a rms roughness of $1.4 \mathrm{~nm}$ for a $5 \mu \mathrm{m} \times 5 \mu \mathrm{m}$ area). Thus, we believe that the roughness of the film is not responsible for the observed decrease of $T_{c}$.

Figure 4(c) shows the $T_{c b} / T_{c s}$ of the $\mathrm{Nb} / \mathrm{SmB}_{6}$ bilayers as a function of $d_{\mathrm{Nb}}$. As a comparison, one bilayer is made with an ex situ interface: following the deposition of $\mathrm{SmB}_{6}$, it is exposed to air before the $\mathrm{Nb}$ layer is deposited. This ex situ sample shows a much higher $T_{c b} / T_{c s}$ (i.e., higher $T_{c}$ ) than the corresponding in situ bilayer. The proximity effect is very sensitive to the nature of the interface, and degradation of the topmost surface layer of $\mathrm{SmB}_{6}$ (e.g., due to oxidation) can reduce the boundary transparency, resulting in reduction of the proximity effect. This result demonstrates that in situ formed multilayers with pristine interfaces are critical for establishing the proximity effect at the interface of $\mathrm{SmB}_{6}$ and a superconductor.

To evaluate $\xi_{\mathrm{SmB}_{6}}$, we perform a fitting process based on the expression discussed above. Using measured values of $\rho_{\mathrm{Nb}}=8.5 \times 10^{-6} \Omega \mathrm{cm}$, the mean-free path $(l)$ of $\mathrm{Nb}$ was estimated from $\rho_{\mathrm{Nb}} l=3.75 \times 10^{-6} \mu \Omega \mathrm{cm}^{2}$ [54] (i.e., $l=4.4 \mathrm{~nm})$ and we obtain $\xi_{\mathrm{Nb}}=0.852\left(\xi_{\mathrm{Nb}(\text { bulk })} l\right)^{1 / 2}=$ $11 \mathrm{~nm}$, where $\xi_{\mathrm{Nb} \text { (bulk) }}$ is the known value of coherence length of bulk $\mathrm{Nb}\left(\xi_{\mathrm{Nb} \text { (bulk) }}=38 \mathrm{~nm}\right)$ [55]. The thickness of the surface conducting channel of $\mathrm{SmB}_{6}$ is evaluated above from our transport result $\left(t_{\text {surface }}=6 \mathrm{~nm}\right)$, and the resistivity of the surface state is calculated to be $9.4 \times$ $10^{-5} \Omega \mathrm{cm}\left(=\rho_{\mathrm{SmB}_{6}}\right)$ at $2 \mathrm{~K}$, based on the above measured sheet conductance $\left(\rho=2 t_{\text {surface }} / G_{\square}\right)$. The only unknown parameter is $\xi_{\mathrm{SmB}_{6}}$. The fitting result is shown as a red solid line in Fig. 4(c), and we arrive at $\xi_{\mathrm{SmB}_{6}}$ of $8.7 \mathrm{~nm}$. To calculate $v_{F}, \xi=\left(v_{F} \hbar l / 6 \pi k_{B} T\right)^{1 / 2}$ is used [56], where $l$ of the $\mathrm{SmB}_{6}$ thin film was assumed to be the grain size of the $\mathrm{SmB}_{6}$ film, $\approx 4 \mathrm{~nm}$ [37], and we obtain $v_{F} \approx 10^{5} \mathrm{~m} / \mathrm{s}$ at $2 \mathrm{~K}$ for $\mathrm{SmB}_{6}$. This $v_{F}$ is comparable to the value obtained from the transport study above $\left(v_{F}=1.6 \times 10^{5} \mathrm{~m} / \mathrm{s}\right)$, which implies that the observed superconducting proximity effect can be attributed to the surface state of $\mathrm{SmB}_{6}$ thin film. As a comparison, we also carry out a fit using the entire bulk of the $\mathrm{SmB}_{6}$ thin film (even though we show above that the bulk of the film is insulating): in this case, the fit does not provide a $v_{F}$ value consistent with the $v_{F}$ value obtained from the transport study. We also note that our bilayer fabrication method in an ultrahigh vacuum process precludes any degradation or contamination as the origin of the metallic surface state.

The proximity effect is expected to persist for bilayers with thinner $\mathrm{SmB}_{6}$ (than $50 \mathrm{~nm}$ discussed above), and we expect it to be suppressed only when the $\mathrm{SmB}_{6}$ thickness approaches its normal coherence length. As can be seen in Fig. 4(c), the bilayers with $25-\mathrm{nm} \mathrm{SmB}_{6}$ show the same trend as the $50-\mathrm{nm} \mathrm{SmB}_{6}$ bilayer series. On the other hand, when the $\mathrm{SmB}_{6}$ layer is $10 \mathrm{~nm}$, the proximity effect is suppressed, resulting in a much higher $T_{c b} / T_{c s}$ for this data point. These results provide collective evidence that the observed systematic drop in $T_{c}$ as function of the $\mathrm{Nb}$ layer thickness is indeed due to the superconducting proximity effect.

The value of $v_{F}$ and the effective mass of quasiparticles in the surface state of $\mathrm{SmB}_{6}$ have been the subjects of much debate. Early theoretical calculations had suggested the presence of relatively heavy Dirac quasiparticles with small $v_{F}\left(\sim 10^{3} \mathrm{~m} / \mathrm{s}\right)[26,57]$ in the topologically protected surface state, while recent APRES and quantum oscillation studies have pointed to light quasiparticles with large $v_{F}$ of $>10^{4} \mathrm{~m} / \mathrm{s}[19,32,33]$. To resolve the discrepancy, Alexandrov et al. have recently proposed that Kondo breakdown at the surface of $\mathrm{SmB}_{6}$ could release unquenched moments at the surface, causing the Dirac point to shift down into the valence band and give rise to large $v_{F}$ values [58]. We examine the $\mathrm{Sm}$ valence state in the surface of our $\mathrm{SmB}_{6}$ thin films using x-ray photoemission spectroscopy at room temperature and find that it is similar to that of $\mathrm{SmB}_{6}$ single crystals, which is $~ 2.7$ [59] (see Supplemental Material [43]). Therefore, we exclude chemical extrinsic effects as the origin of the large $v_{F}$ value observed here. We believe our high $v_{F}$ value is also due to the Kondo breakdown effect [58]. We note that $t_{\text {surface }}$ of $\approx 6 \mathrm{~nm}$ found in this study is consistent with the thickness predicted with the Kondo breakdown effect [58]. It has been reported that $\mathrm{SmB}_{6}$ has three Dirac cones, each with its own slightly different value of $v_{F}[32,60]$. The measured $v_{F}$ in this study is, therefore, expected to be the average $v_{F}$ of the three Dirac cones.

With the $\xi_{\mathrm{SmB}_{6}}$ obtained from the fitting above, we are able to evaluate $\gamma$, which is a measure of the strength of the superconducting proximity effect. The values of $\gamma$ for in situ and ex situ bilayers are $7.9 \times 10^{-2}$ and $1.0 \times 10^{-2}$, respectively. This implies that the strength of the proximity effect of the in situ sample is roughly 8 times larger than that of the ex situ sample. The strength of the superconducting proximity effect is naturally one of the most important factors in superconductor-normal-superconductor Josephson junctions, and it directly affects the $I_{C} R_{N}$ product. It is interesting to note that significantly reduced $I_{C} R_{N}$ products, presumably due to lack of the pristine surface of the TI during the fabrication process, have been observed in various studies on chalcogenide-TI-based Josephson junctions [5,6]. Specifically, the observed $I_{C} R_{N}$ products in such studies are typically around $20 \mu \mathrm{V}$, which is far below the theoretical value, i.e., $I_{C} R_{N} \sim \pi \Delta(0) / 2 e \approx 4.7 \mathrm{mV}$ (for $\mathrm{Nb}$ ), for junctions in the clean limit $[61,62]$. Higher $I_{C} R_{N}$ product junctions (and, in particular, high $I_{C}$ junctions) are always desirable for investigating novel quantum phenomenainvolving coherence of superconductivity, where a signature of such phenomena might appear in the form of 
small modulation in the critical current. The present study shows that the strength of proximity effect in the bilayer with an in situ interface is nearly an order of magnitude higher than that with an ex situ interface. We expect that with identical geometric dimensions, junctions fabricated by in situ interface may display a substantially larger $I_{C} R_{N}$ than junctions fabricated with an ex situ interface. Thus, we demonstrate an important prerequisite for attaining a high $I_{C} R_{N}$ junction. A nonideal S-N interface, such as one with a thin tunnel barrier (formed due to an ex situ process), can result in scattering at the interface leading to decoherence $[7,63]$. This may be the reason why the proposed signatures of Majorana fermions have not been observed in Josephson junctions to date.

\section{CONCLUSIONS}

In summary, thickness-independent behavior in the transport properties is observed in ultrathin $\mathrm{SmB}_{6}$ films, and the thickness of the surface state is deduced to be $\approx 6 \mathrm{~nm}$. We provide first direct evidence of the superconducting proximity effect in the surface state of $\mathrm{SmB}_{6}$ through pristine interfaces with in situ deposited $\mathrm{Nb}$ layers. The Fermi velocity values of the surface state obtained from the transport measurements and the proximity effect are in good agreement with each other. The present work lays the groundwork for fabricating $\mathrm{SmB}_{6}$ thin-film-based multilayers and devices for investigating quantum phenomena including Majorana fermion excitations.

\section{ACKNOWLEDGMENTS}

The authors would like to acknowledge Victor Galitski for valuable discussions. This work was supported by AFOSR (Grant No. FA9550-14-10332) and NSF (Grant No. DMR-1410665). It was also supported by the Maryland NanoCenter.

[1] A. C. Potter and P. A. Lee, Engineering a $p+i p$ Superconductor: Comparison of Topological Insulator and Rashba Spin-Orbit-Coupled Materials, Phys. Rev. B 83, 184520 (2011).

[2] X.-L. Qi, T. L. Hughes, and S.-C. Zhang, Chiral Topological Superconductor from the Quantum Hall State, Phys. Rev. B 82, 184516 (2010).

[3] X.-L. Qi, T. L. Hughes, and S.-C. Zhang, Topological Field Theory of Time-Reversal Invariant Insulators, Phys. Rev. B 78, 195424 (2008).

[4] J. Moore, Extraordinary Josephson Junction, Physics 5, 84 (2012).

[5] M. Veldhorst et al., Josephson Supercurrent through a Topological Insulator Surface State, Nat. Mater. 11, 417 (2012).

[6] J. R. Williams, A. J. Bestwick, P. Gallagher, S. S. Hong, Y. Cui, A.S. Bleich, J. G. Analytis, I. R. Fisher, and
D. Goldhaber-Gordon, Unconventional Josephson Effect in Hybrid Superconductor-Topological Insulator Devices, Phys. Rev. Lett. 109, 056803 (2012).

[7] L. Fu and C. L. Kane, Superconducting Proximity Effect and Majorana Fermions at the Surface of a Topological Insulator, Phys. Rev. Lett. 100, 096407 (2008).

[8] F. Yang et al., Proximity-Effect-Induced Superconducting Phase in the Topological Insulator, Phys. Rev. B 86, 134504 (2012).

[9] C. Nayak, S. H. Simon, A. Stern, M. Freedman, and S. Das Sarma, Non-Abelian Anyons and Topological Quantum Computation, Rev. Mod. Phys. 80, 1083 (2008).

[10] L. Galletti et al., Influence of Topological Edge States on the Properties of $\mathrm{Al} / \mathrm{Bi}_{2} \mathrm{Se}_{3} / \mathrm{Al}$ Hybrid Josephson Devices, Phys. Rev. B 89, 134512 (2014).

[11] B. Sacépé, J. B. Oostinga, J. Li, A. Ubaldini, N. J. G. Couto, E. Giannini, and A. F. Morpurgo, Gate-Tuned Normal and Superconducting Transport at the Surface of a Topological Insulator, Nat. Commun. 2, 575 (2011).

[12] M. Snelder et al., Josephson Supercurrent in a Topological Insulator without a Bulk Shunt, Supercond. Sci. Technol. 27, 104001 (2014).

[13] G. Koren and T. Kirzhner, Zero-Energy Bound States in Tunneling Conductance Spectra at the Interface of an Wave Superconductor and a Topological Insulator in $\mathrm{NbN} / \mathrm{Bi}_{2} \mathrm{Se}_{3} / \mathrm{Au}$ Thin-Film Junctions, Phys. Rev. B 86, 144508 (2012).

[14] Y. Tanaka, T. Yokoyama, and N. Nagaosa, Manipulation of the Majorana Fermion, Andreev Reflection, and Josephson Current on Topological Insulators, Phys. Rev. Lett. 103, 107002 (2009).

[15] G. Koren, Proximity Effects at the Interface of a Superconductor and a Topological Insulator in $\mathrm{NbN}-\mathrm{Bi}_{2} \mathrm{Se}_{3}$ Thin Film Bilayers, Supercond. Sci. Technol. 28, 025003 (2015).

[16] A. A. Golubov, M. Y. Kupriyanov, V. F. Lukichev, and A. A. Orlikovskii, Mikroelektronika 12, 355 (1983) [CriticalTemperature of a SN Sandwich, Sov. J. Microelectronics 12, 191 (1984)].

[17] M. Dzero, J. Xia, V. Galitski, and P. Coleman, Topological Kondo Insulators, Annu. Rev. Condens. Matter Phys. 7, 249 (2016).

[18] J. W. Allen, B. Batlogg, and P. Wachter, Large LowTemperature Hall Effect and Resistivity in Mixed-Valent $\mathrm{SmB}_{6}$, Phys. Rev. B 20, 4807 (1979).

[19] G. Li et al., Two-Dimensional Fermi Surfaces in Kondo Insulator $\mathrm{SmB}_{6}$, Science 346, 1208 (2014).

[20] D. J. Kim, S. Thomas, T. Grant, J. Botimer, Z. Fisk, and J. Xia, Surface Hall Effect and Nonlocal Transport in $\mathrm{SmB}_{6}$ : Evidence for Surface Conduction, Sci. Rep. 3, 3150 (2013).

[21] S. Rößler, T.-H. Jang, D.-J. Kim, L. H. Tjeng, Z. Fisk, F. Steglich, and S. Wirth, Hybridization Gap and Fano Resonance in $\mathrm{SmB}_{6}$, Proc. Natl. Acad. Sci. U.S.A. 111, 4798 (2014).

[22] W. A. Phelan, S. M. Koohpayeh, P. Cottingham, J. W. Freeland, J. C. Leiner, C. L. Broholm, and T. M. McQueen, Correlation between Bulk Thermodynamic Measurements and the Low-Temperature-Resistance Plateau in $\mathrm{SmB}_{6}$, Phys. Rev. X 4, 031012 (2014). 
[23] X. Zhang, N. P. Butch, P. Syers, S. Ziemak, R. L. Greene, and J. Paglione, Hybridization, Inter-ion Correlation, and Surface States in the Kondo Insulator $\mathrm{SmB}_{6}$, Phys. Rev. X 3, 011011 (2013).

[24] P. Syers, D. Kim, M. S. Fuhrer, and J. Paglione, Tuning Bulk and Surface Conduction in the Proposed Topological Kondo Insulator $\mathrm{SmB}_{6}$, Phys. Rev. Lett. 114, 096601 (2015).

[25] W. Ruan, C. Ye, M. Guo, F. Chen, X. Chen, G.-M. Zhang, and Y. Wang, Emergence of a Coherent In-Gap State in the $\mathrm{SmB}_{6}$ Kondo Insulator Revealed by Scanning Tunneling Spectroscopy, Phys. Rev. Lett. 112, 136401 (2014).

[26] V. Alexandrov, M. Dzero, and P. Coleman, Cubic Topological Kondo Insulators, Phys. Rev. Lett. 111, 226403 (2013).

[27] Z.-H. Zhu, A. Nicolaou, G. Levy, N. P. Butch, P. Syers, X. F. Wang, J. Paglione, G. A. Sawatzky, I. S. Elfimov, and A. Damascelli, Polarity-Driven Surface Metallicity in $\mathrm{SmB}_{6}$, Phys. Rev. Lett. 111, 216402 (2013).

[28] C. Cooley, M. C. Aronson, Z. Fisk, and P. C. Canfield, $\mathrm{SmB}_{6}$-Kondo Insulator or Exotic Metal, Phys. Rev. Lett. 74, 1629 (1995).

[29] N. Wakeham, Y. Q. Wang, Z. Fisk, F. Ronning, and J. D. Thompson, Surface State Reconstruction in Ion-Damaged $\mathrm{SmB}_{6}$, Phys. Rev. B 91, 085107 (2015).

[30] B. Roy, J. D. Sau, M. Dzero, and V. Galitski, Surface Theory of a Family of Topological Kondo Insulators, Phys. Rev. B 90, 155314 (2014).

[31] S. Wolgast, Ç. Kurdak, K. Sun, J. W. Allen, D.-J. Kim, and Z. Fisk, Low-Temperature Surface Conduction in the Kondo Insulator $\mathrm{SmB}_{6}$, Phys. Rev. B 88, 180405 (2013).

[32] J. Jiang et al., Observation of Possible Topological In-Gap Surface States in the Kondo Insulator $\mathrm{SmB}_{6}$ by Photoemission, Nat. Commun. 4, 3010 (2013).

[33] M. Neupane et al., Surface Electronic Structure of the Topological Kondo-Insulator Candidate Correlated Electron System $\mathrm{SmB}_{6}$, Nat. Commun. 4, 2991 (2013).

[34] N. Xu et al., Direct Observation of the Spin Texture in $\mathrm{SmB}_{6}$ as Evidence of the Topological Kondo Insulator, Nat. Commun. 5, 4566 (2014).

[35] D. J. Kim, J. Xia, and Z. Fisk, Topological Surface State in the Kondo Insulator Samarium Hexaboride, Nat. Mater. 13, 466 (2014).

[36] J. Yong, Y. Jiang, X. Zhang, J. Shin, I. Takeuchi, and R. L. Greene, Magnetotransport in Nanocrystalline $\mathrm{SmB}_{6}$ Thin Films, AIP Adv. 5, 077144 (2015).

[37] J. Yong, Y. Jiang, D. Usanmaz, S. Curtarolo, X. Zhang, L. Li, X. Pan, J. Shin, I. Takeuchi, and R. L. Greene, Robust Topological Surface State in Kondo Insulator $\mathrm{SmB}_{6}$ Thin Films, Appl. Phys. Lett. 105, 222403 (2014).

[38] Y. Nakajima, P. Syers, X. Wang, R. Wang, and J. Paglione, One-Dimensional Edge State Transport in a Topological Kondo Insulator, Nat. Phys. 12, 213 (2016).

[39] M. M. Yee, Y. He, A. Soumyanarayanan, D.-J. Kim, Z. Fisk, and J. E. Hoffman, Imaging the Kondo Insulating Gap on $\mathrm{SmB}_{6}$, arXiv:1308.1085v2.

[40] P. Hlawenka et al., Samarium Hexaboride: A Trivial Surface Conductor, arXiv:1502.01542.
[41] B.S. Tan et al., Unconventional Fermi Surface in an Insulating State, Science 349, 287 (2015).

[42] J. D. Denlinger et al., Consistency of Photoemission and Quantum Oscillations for Surface States of $\mathrm{SmB}_{6}$, arXiv:1601.07408v1.

[43] See Supplemental Material at http://link.aps.org/ supplemental/10.1103/PhysRevX.6.031031 for sheet carrier concentration and mobility, and $\mathrm{Sm}$ valence state examined by X-ray photoemission spectroscopy.

[44] B. Xia, P. Ren, A. Sulaev, P. Liu, S.-Q. Shen, and L. Wang, Indications of Surface-Dominated Transport in Single Crystalline Nanoflake Devices of Topological Insulator $\mathrm{Bi}_{1.5} \mathrm{Sb}_{0.5} \mathrm{Te}_{1.8} \mathrm{Se}_{1.2}$, Phys. Rev. B 87, 085442 (2013).

[45] N. Xu et al., Exotic Kondo Crossover in a Wide Temperature Region in the Topological Kondo Insulator $\mathrm{SmB}_{6}$ Revealed by High-Resolution ARPES, Phys. Rev. B 90, 085148 (2014).

[46] J. D. Denlinger, J. W. Allen, J.-S. Kang, K. Sun, J.-W. Kim, J. H. Shim, B. I. Min, D.-J. Kim, and Z. Fisk, Temperature Dependence of Linked Gap and Surface State Evolution in the Mixed Valent Topological Insulator $\mathrm{SmB}_{6}$, arXiv:1312.6637.

[47] J. Linder, T. Yokoyama, and A. Sudbø, Anomalous Finite Size Effects on Surface States in the Topological Insulator $\mathrm{Bi}_{2} \mathrm{Se}_{3}$, Phys. Rev. B 80, 205401 (2009).

[48] J. Zhang, J. Yong, I. Takeuchi, R. L. Greene, and R. D. Averitt, Ultrafast Terahertz Spectroscopy Study of Kondo Insulating Thin Film $\mathrm{SmB}_{6}$ : Evidence for an Emergent Surface State, arXiv:1509.04688.

[49] A. A. Golubov, Proximity Effect in Dirty N/S Multilayers, Proc. SPIE 2157, 353 (1994).

[50] K. D. Usadel, Generalized Diffusion Equation for Superconducting Alloys, Phys. Rev. Lett. 25, 507 (1970).

[51] H. Onoe, A. Sumiyama, M. Nakagawa, and T. Oda, Superconducting Proximity Effect in Au of Au-Clad $\mathrm{Nb}$ Wire in the Clean Limit, J. Phys. Soc. Jpn. 64, 2138 (1995).

[52] J. Kim, Y.-J. Doh, K. Char, H. Doh, and H.-Y. Choi, Proximity Effect in $\mathrm{Nb} / \mathrm{Au} / \mathrm{CoFe}$ Trilayers, Phys. Rev. B 71, 214519 (2005).

[53] M. S. M. Minhaj, S. Meepagala, J. T. Chen, and L. E. Wenger, Thickness Dependence on the Superconducting Properties of Thin Nb Films, Phys. Rev. B 49, 15235 (1994).

[54] A. F. Mayadas, R. B. Laibowitz, and J. J. Cuomo, Electrical Characteristics of rf-Sputtered Single-Crystal Niobium Films, J. Appl. Phys. 43, 1287 (1972).

[55] C. Delacour, L. Ortega, M. Faucher, T. Crozes, T. Fournier, B. Pannetier, and V. Bouchiat, Persistence of Superconductivity in Niobium Ultrathin Films Grown on R-Plane Sapphire, Phys. Rev. B 83, 144504 (2011).

[56] P. Seidel, Applied Superconductivity: Handbook on Devices and Applications (John Wiley \& Sons, New York, 2015).

[57] M. Dzero, K. Sun, P. Coleman, and V. Galitski, Theory of Topological Kondo Insulators, Phys. Rev. B 85, 045130 (2012).

[58] V. Alexandrov, P. Coleman, and O. Erten, Kondo Breakdown in Topological Kondo Insulators, Phys. Rev. Lett. 114, 177202 (2015). 
[59] J. N. Chazalviel, M. Campagna, and G. K. Wertheim, Study of Valence Mixing in $\mathrm{SmB}_{6}$ by X-Ray Photoelectron Spectroscopy, Phys. Rev. B 14, 4586 (1976).

[60] F. Lu, J. Z. Zhao, H. Weng, Z. Fang, and X. Dai, Correlated Topological Insulators with Mixed Valence, Phys. Rev. Lett. 110, 096401 (2013).

[61] M. Tinkham, Introduction to Superconductivity (McGrawHill, New York, 1996).
[62] C. Kittel, Introduction to Solid State Physics (Wiley, New York, 2005).

[63] A. Tesauro, A. Aurigemma, C. Cirillo, S. L. Prischepa, M. Salvato, and C. Attanasio, Interface Transparency and Proximity Effect in $\mathrm{Nb} / \mathrm{Cu}$ Triple Layers Realized by Sputtering and Molecular Beam Epitaxy, Supercond. Sci. Technol. 18, 1 (2005). 